\title{
Indistinguishable and efficient single photons from a quantum dot in a planar nanobeam waveguide
}

\author{
Gabija Kiršanske,${ }^{1}$ Henri Thyrrestrup, ${ }^{1}$ Raphaël S. Daveau, ${ }^{1}$ Chris L. Dreeßen, ${ }^{1}$ Tommaso Pregnolato, ${ }^{1}$ Leonardo Midolo, ${ }^{1}$ \\ Petru Tighineanu, ${ }_{1}^{1}$ Alisa Javadi, ${ }^{1}$ Søren Stobbe, ${ }^{1}$ Rüdiger Schott, ${ }^{2}$ Arne Ludwig, ${ }^{2}$ Andreas D. Wieck, ${ }^{2}$ Suk In Park, ${ }^{3}$ \\ Jin D. Song, ${ }^{3}$ Andreas V. Kuhlmann, ${ }^{4}$ Immo Söllner, ${ }^{4}$ Matthias C. Löbl, ${ }^{4}$ Richard J. Warburton, ${ }^{4}$ and Peter Lodahl ${ }^{1}$ \\ ${ }^{1}$ Niels Bohr Institute, University of Copenhagen, Blegdamsvej 17, DK-2100 Copenhagen, Denmark \\ ${ }^{2}$ Lehrstuhl für Angewandte Festkörperphysik, Ruhr-Universität Bochum, Universitätsstrasse 150, D-44780 Bochum, Germany \\ ${ }^{3}$ Center for Opto-Electronic Convergence Systems, Korea Institute of Science and Technology, Seoul 136-791, Korea \\ ${ }^{4}$ Department of Physics, University of Basel, Klingelbergstrasse 82, CH-4056 Basel, Switzerland \\ (Received 27 January 2017; revised manuscript received 2 October 2017; published 19 October 2017)
}

\begin{abstract}
We demonstrate a high-purity source of indistinguishable single photons using a quantum dot embedded in a nanophotonic waveguide. The source features a near-unity internal coupling efficiency and the collected photons are efficiently coupled off chip by implementing a taper that adiabatically couples the photons to an optical fiber. By quasiresonant excitation of the quantum dot, we measure a single-photon purity larger than $99.4 \%$ and a photon indistinguishability of up to $94 \pm 1 \%$ by using $p$-shell excitation combined with spectral filtering to reduce photon jitter. A temperature-dependent study allows pinpointing the residual decoherence processes, notably the effect of phonon broadening. Strict resonant excitation is implemented as well as another means of suppressing photon jitter, and the additional complexity of suppressing the excitation laser source is addressed. The paper opens a clear pathway towards the long-standing goal of a fully deterministic source of indistinguishable photons, which is integrated on a planar photonic chip.
\end{abstract}

DOI: 10.1103/PhysRevB.96.165306

\section{INTRODUCTION}

A truly on-demand source of coherent single photons is the essential quantum hardware behind many photonic quantuminformation applications including device-independent quantum cryptography [1,2] and quantum simulations [3] or, more daring, a full-scale photonic quantum computer [4,5] or a photonic quantum network [6,7]. The demands on source performance depend on the actual application in mind, and currently the first proof-of-concept demonstrations are emerging. At present the state of the art in the field is the achievement of ten-photon entanglement [8] and quantum simulations with three to four photons $[9,10]$. A highly promising route of extending beyond this performance applies self-assembled quantum dots (QDs) as single-photon emitters [11] embedded in photonic nanostructures to enhance light-matter interaction [12]. This platform has matured significantly in recent years [12-26]. So far, much progress has been made on micropillar cavities and nanowires where the collected photons are coupled vertically out of the structure in a confocal microscopy setup. Planar nanophotonic waveguides offer the opportunity of increasing the single-photon coupling efficiency to near unity [19]. Importantly, the waveguide-integrated platform provides a route to on-chip photonic quantum-information processing. It remains to be demonstrated that highly coherent single photons can be generated on this platform, where the presence of surfaces near the QD may lead to decoherence [27]. Indeed the thin $(\sim 160 \mathrm{~nm})$ and narrow $(\sim 300 \mathrm{~nm})$ waveguide structures imply that the embedded QDs are unavoidably close to doped semiconductor material and semiconductor-air interfaces.

We present the demonstration of a highly coherent singlephoton source based on an electrically controlled QD integrated in a high-efficiency nanophotonic waveguide. Pulsed quasiresonant and strict resonant excitation of the QD in the waveguide is applied to deterministically operate the singlephoton source, whereby the indistinguishability of the emitted photons can be directly determined as opposed to a continuous- wave excitation experiment [28,29]. Single-photon purity exceeding $99.4 \%$ is demonstrated together with a two-photon interference visibility of $62 \%$ for quasiresonant excitation. These values are limited primarily by photon jitter associated with the relaxation from the excited $p$ shell and into the ground state. On a different sample, we show that the spectral filtering allows suppressing photon jitter significantly and observe an indistinguishability as high as $94 \pm 1 \%$. Strict resonant excitation is implemented as an alternative method of suppressing photon jitter without the need to spectrally filter the output, while instead the efficient suppression of the pump light is required. Finally, it is demonstrated how the emitted photons can efficiently be coupled off chip to an optical fiber by the implementation of a tapered waveguide section. Our paper paves the way for a fully deterministic source of indistinguishable photons for scalable quantum-information processing applications.

The paper is organized as follows. In Sec. II the structure design, the experimental methods, and the results of the quasiresonant indistinguishability measurements are presented. In Sec. III we discuss the outcoupling taper design and the results of the source efficiency characterization performed. In Sec. IV we present the Hong-Ou-Mandel (HOM) experiment results on a strictly resonantly excited QD embedded in a nanobeam waveguide terminated by a grating outcoupler. We present the conclusions in Sec. V. In Appendix A we discuss the fitting routine allowing for reliable extraction of photon indistinguishability from a pulsed HOM experiment. Details on the analysis of the resonance fluorescence HOM data with cw background are included in Appendix B.

\section{PHOTON INDISTINGUISHABILITY UNDER QUASIRESONANT EXCITATION}

\section{A. Structure design: Device A}

The investigated device (device A) consists of a suspended waveguide with integrated metal contacts in order to apply an external bias across the InGaAs QD to tune and stabilize 
the transition. The 300-nm-wide suspended waveguides are fabricated on a $p-i$ - $n$ GaAs wafer [see Fig. 1(c) for the layer structure] using electron-beam lithography followed by dry and wet etching processes, following the methods described in Ref. [30]. Figure 1(d) shows a scanning-electron microscope image of the samples terminated with a taper, e.g., device A, where a precise cleaving method is implemented to obtain tapers protruding from the edge of the sample such that the optical mode in the taper can freely expand without reflecting from the device substrate. The internal efficiency of the source is quantified by the $\beta$ factor, which is the single-photon coupling efficiency into the waveguide mode [12]. Figure 1(b) displays the spatial dependance of the simulated $\beta$ factor in the waveguide for the two relevant dipole orientations. At the center of the waveguide the optical transition polarized along the $y$ dipole couples to the main waveguide mode with $96 \%$ efficiency, whereas the orthogonal dipole is polarization filtered in the structure. We implement a way to outcouple photons from a waveguide on a photonic chip directly to a single-mode fiber, thus discarding the need for a confocal setup. Further details on the taper design are discussed in Sec. III.

\section{B. Experimental conditions}

For the optical measurements under quasiresonant excitation, device $\mathrm{A}$ is mounted on a three-axis piezoelectric stage in a liquid-helium bath cryostat and cooled to temperatures between 4.2 and $26.4 \mathrm{~K}$ [see Fig. 1(f)]. The QD is excited with a picosecond-pulsed Ti:sapphire laser at a repetition rate of $76 \mathrm{MHz}$ and focused through a microscope objective with $\mathrm{NA}=0.55$. The laser polarization is set to the orientation resulting in the highest count rate of the emitted photons. A delay is introduced in the laser path to allow for a periodic excitation with double pulses delivered with a time separation of $2.7 \mathrm{~ns}$. The emitted photons are collected by a lensed single-mode fiber mounted on a separate stack of piezoelectric stages allowing for the taper-fiber spatial alignment and guided to a detection setup. Polarization scrambling in the single-mode fibers is corrected by the polarization optics after the lensed fiber. A grating filtering setup is implemented (spectral resolution, $70 \mathrm{pm} / 25 \mathrm{GHz}$; transmission throughput, $27 \%$ ) in order to remove the phonon sidebands of the single photons, whereby only the spectrally narrow zero-phonon line is analyzed. The emitted photons are characterized in a HOM interferometer [31] illustrated in Fig. 1(f). Here the spectrally filtered photons are sent to an asymmetric Mach-Zehnder interferometer designed to record the quantum interference of subsequently emitted photons. The photon indistinguishability is obtained from the two-photon autocorrelation function constructed by correlating two single-photon detectors.

\section{Single-photon purity}

The purity of single-photon emission can be characterized in a Hanbury Brown-Twiss measurement of the photon autocorrelation function. The representative experimental data

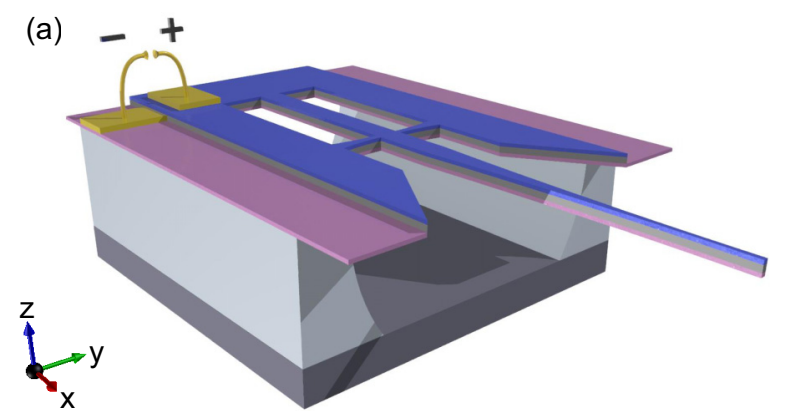

(b)
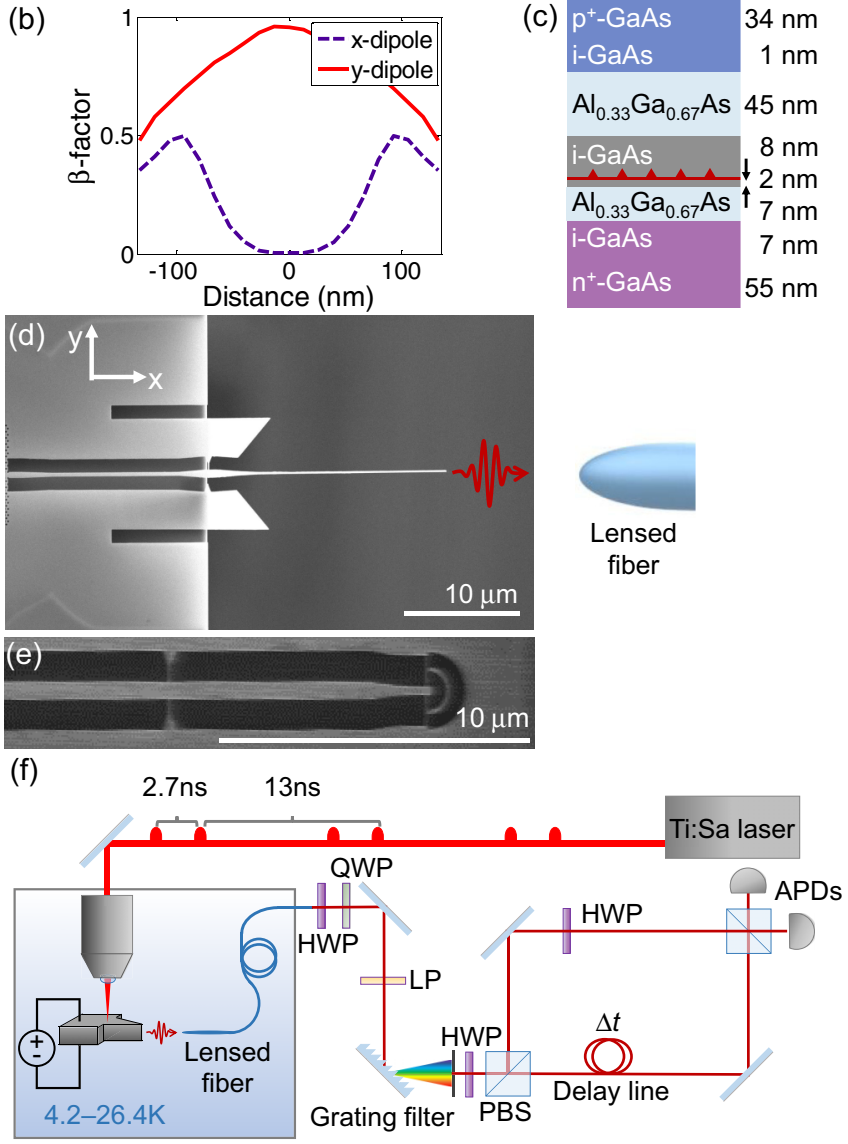

FIG. 1. Two-photon interference experiment on a QD embedded in a tapered nanobeam waveguide. (a) Sketch of the device. (b) Calculated $\beta$ factor for the two dipole orientations along the $x$ and $y$ axes of the nanobeam waveguide as a function of distance from the waveguide center along the $y$ axis. (c) Layer structure of the wafer used for sample fabrication, where the intrinsic layer in the middle of a $p$ - $i-n$ diode contains InGaAs QDs. (d) Scanning-electron micrograph of the waveguide sample with adiabatically tapered outcouplers. The emitted photons are collected by a lensed single-mode fiber. (e) Scanning-electron micrograph of the waveguide sample terminated by a circular grating outcoupler guiding the emitted photons out of plane. (f) Schematics of the experimental setup to measure the indistinguishability of single photons consecutively emitted with variable time delay $\Delta t$. The photoluminescence of the QD is tuned via the applied electric field. The emitted photons are collected with a lensed fiber and sent to a HOM interferometer for correlation measurements. HWP, half-wave plate; QWP, quarter-wave plate; LP, linear polarizer; PBS, polarizing beam splitter; APDs, avalanche photodiodes. 

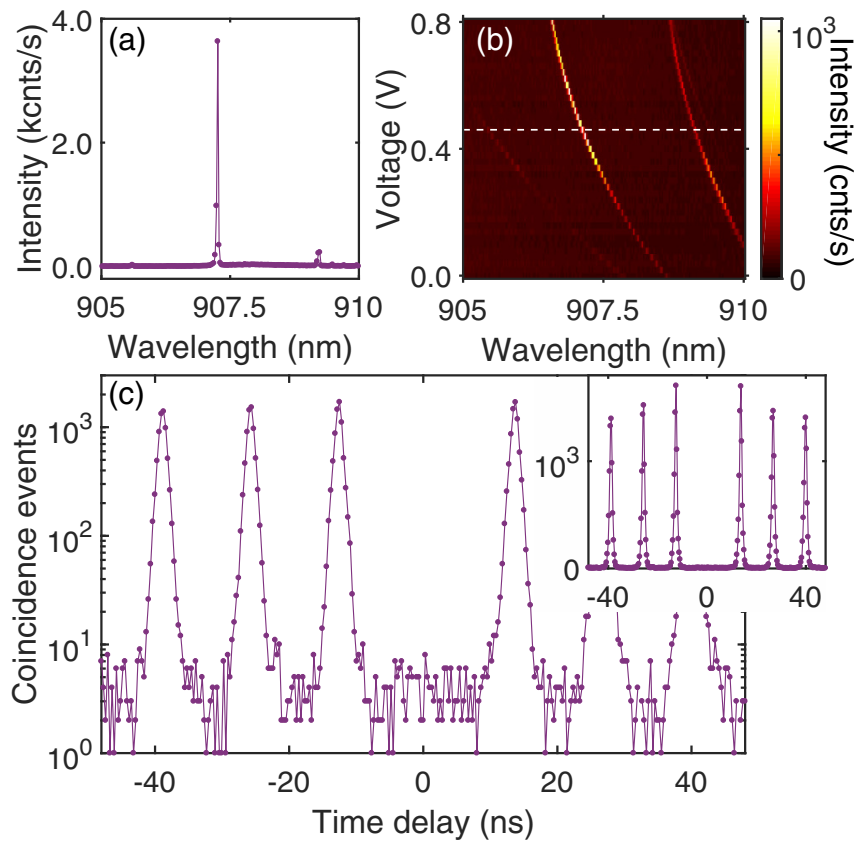

FIG. 2. Single-photon emission from an electrically controlled QD in device A under pulsed $p$-shell excitation at $4.2 \mathrm{~K}$. (a) Photoluminescence spectrum of the QD emitting at $907.4 \mathrm{~nm}$ at $0.46-\mathrm{V}$ bias [indicated by the white dashed line in (b)]. The width of the emission line is limited by the resolution of the spectrometer. (b) Photoluminescence map of the QD vs the applied voltage measured at an excitation level of $80 \%$ of the saturation power. An extra line from the same QD is identified at a lower voltage and the pair most likely is the $X^{0}$ and $X^{+}$transitions. The circularly polarized $X^{+}$couples less efficiently to the nanobeam waveguide resulting in a weaker optical signature than $X^{0}$. (c) Intensity-correlation histogram from the QD under $p$-shell excitation on a logarithmic scale. Nearly ideal single-photon emission is demonstrated by the vanishing multiphoton probability at zero time delay $g^{2}(0)<0.006$. The inset shows the data in (c) on a linear scale.

reported here were recorded on device A. Figure 2(a) shows a typical emission spectrum for a QD quasiresonantly excited at a wavelength of $888 \mathrm{~nm}$, which may correspond to either $p$ shell or LO-phonon assisted excitation. A constant bias voltage of $0.46 \mathrm{~V}$ is applied to the QD in order to achieve the highest photon count rate, as deduced from the photoluminescencevoltage map, shown in Fig. 2(b). The measured autocorrelation function of $g^{2}(0)<0.006$ corresponds to an excellent singlephoton purity larger than $99.4 \%$ [see Fig. 2(c)], where $g^{2}(0)$ is obtained as the ratio of integrated counts of the fitted peak centered at zero time delay relative to the peak area in the uncorrelated limit of a long time delay. This accounts for the weak blinking of emission (at a preparation efficiency of 75\%) found on a time scale of about $40 \mathrm{~ns}[37,38]$.

\section{Two-photon interference}

The indistinguishability of the single photons can be tested by two-photon interference measurements of consecutively emitted photons. Figure 3(a) shows a correlation histogram of the two-photon coincidence events acquired on device A. The five-peak structure of the histogram stems from the arrival
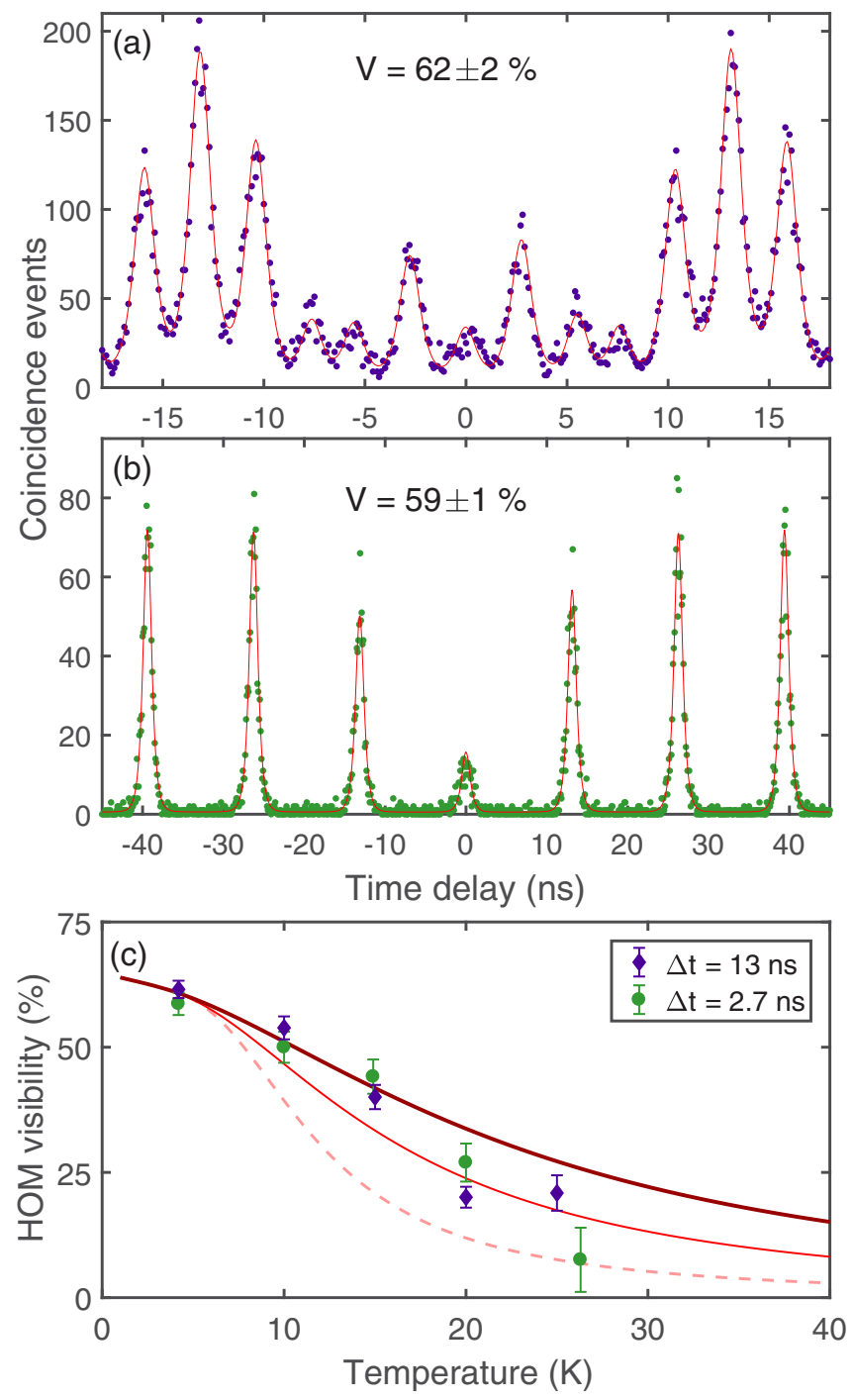

FIG. 3. Recorded correlation histograms in the HOM interferometer of device A for a successive time separation of emitted photons of $2.7 \mathrm{~ns}$ (a) and $13 \mathrm{~ns}$ (b), respectively, measured at $4.2 \mathrm{~K}$ and at $0.8 P_{\text {sat }}$ excitation power. The data points present the raw data without any background correction after integration over 2.5 and $1 \mathrm{~h}$, respectively. We note that the outcoupling and detection efficiency was not optimized in this experiment. The red line displays the modeling of the data. The visibility $V$ is extracted by fitting the data with the convolution of a single-exponential decay (QD decay) and a Voigt function (photodetector response); see Appendix A. (c) Measured temperature dependence of the visibility for the two time separations. The error bars are calculated by error propagation of the fitted parameter errors. The curves are obtained from the theory presented in Ref. [32], and for spherical QDs of radii 2, 3, and $4 \mathrm{~nm}$ $[33,34]$ and corresponding hole (electron) energy splitting between $s$ and $p$ shell of 15,20 , and $30 \mathrm{meV}(30,40$, and $60 \mathrm{meV})[35,36]$ denoted by the dashed, thin solid, and thick solid lines, respectively.

of the photons at different time intervals. The central peak corresponds to the situation where two photons meet up on the beam splitter at the same time and would vanish for completely indistinguishable photons. The degree of indistinguishability is quantified from the visibility $(V)$ in the HOM experiment, which can be extracted from the relative area of the central 
peak compared to neighboring peaks $[13,23]$. We implement a rigorous fitting routine, which takes into account the exponential decay of the emitter, the measured instrument response function, and Poissonian counting statistics. This is essential in order to reliably extract $V$, and is not generally performed in the literature [21,22,25,26,39-42], with the notable exception of Ref. [43]. In Appendix A the details of the data analysis are presented, and it is found that an overestimation of $V$ of up to $30 \%$ may be done if the data analysis often presented in the literature is implemented. Figure 3(a) displays the data for a time delay in between photons of $2.7 \mathrm{~ns}$ where we obtain $V=62 \pm 2 \%$. The experiment was also repeated for a time separation of $13 \mathrm{~ns}$ [see Fig. 3(b)], where we extract $V=59 \pm 1 \%$, which agrees within the error bars of the measurement with the 2.7-ns data.

The residual decoherence processes found in the measurements can be attributed to two different processes: time-jitter induced by the relaxation of the carrier from the quasiresonant excitation to the QD ground state expressed by the rate $\Gamma_{\mathrm{jitter}}$ [44] and temperature-dependent broadening [pure dephasing rate $\Gamma_{\mathrm{ph}}(T)$ ] of the zero-phonon line of the QD due to interaction with phonons [45]. The visibility can be expressed as [44]

$$
V=\frac{\Gamma_{\mathrm{rad}}}{\left[\Gamma_{\mathrm{rad}}+\Gamma_{\mathrm{ph}}(T)\right]\left(1+\Gamma_{\mathrm{rad}} / \Gamma_{\mathrm{jitter}}\right)},
$$

where $\Gamma_{\mathrm{rad}}=2.3 \mathrm{~ns}^{-1}$ is the measured radiative decay rate of the QD. Figure 3(c) shows experimental data of the temperature dependence of the visibility. It is found to decrease significantly with temperature, which is indicative of phonon dephasing. The experimental data are compared to a theoretical model predicting the reduction of indistinguishability with temperature due to the broadening of the zero-phonon line [32] for three indicative sets of parameters [see Fig. 3(c)]. We obtain $\Gamma_{\text {jitter }}=3.7 \mathrm{~ns}^{-1}$ similar to literature reported values [46]. This contribution may be reduced by applying resonant ( $\pi$-pulse) excitation or spectral filtering, as will be demonstrated in Sec. IV. As an important reference point the achievable indistinguishability limitation due to phonons is $V=94 \%$ at $T=4 \mathrm{~K}$, which is consistent with linewidth measurements [47]. Ultimately the indistinguishability will be limited by the increased broadening of the zero-phonon line due to mechanical vibrations predicted in a one-dimensional optical system [32]. This could be improved further by either cooling the sample further down or by implementing Purcell enhancement. For instance, at $T=1 \mathrm{~K}$ the indistinguishability would increase to $98.6 \%$ or alternatively a readily achievable Purcell factor of 10 [12] would lead to $V=98.8 \%$ at $T=4 \mathrm{~K}$. In Sec. IV we demonstrate $\mathrm{V}=94 \%$.

\section{SINGLE-PHOTON SOURCE EFFICIENCY}

\section{A. Efficient outcoupling taper design}

The efficient extraction of the emitted photons is implemented through an adiabatic taper outcoupler, which is presented in the following. The waveguide structures are terminated with a taper outcoupling section inspired by the work of Cohen et al. [48], and adapted to GaAs for a working wavelength of $940 \mathrm{~nm}$. The efficient coupling from a submicrometer sized waveguide to a $5-\mu \mathrm{m}$ single-mode fiber
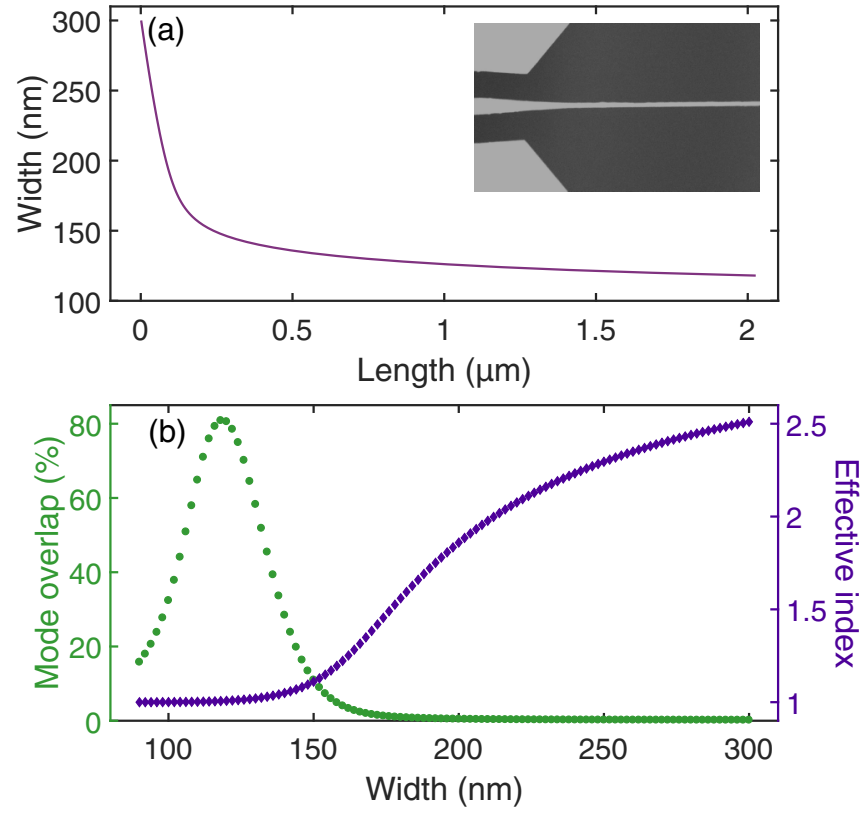

FIG. 4. Taper shape and properties extracted from the finiteelement method simulation of a 160 -nm-thick GaAs waveguide surrounded by air. (a) Taper profile (width as a function of length) obtained from the adiabatic rule with $\alpha=1$ to emphasize the shape. Inset: A fragment of a scanning-electron micrograph of a fabricated taper with $\alpha=10$. (b) Mode overlap between the waveguide mode and a Gaussian distribution of $2.5-\mu \mathrm{m}$ mode-field diameter (green dotted curve). The maximum mode overlap serves as a guideline for the optimal taper width. The effective index as a function of the waveguide width (blue points) illustrates when the optical mode is fully leaked out into air (at a width of $\lesssim 150 \mathrm{~nm}$ ).

core requires a redesign of the two systems to achieve good mode matching. Lensed fibers allow us to reduce the fiber mode typically to a few microns, however the dimensions of the $300 \times 160 \mathrm{~nm}$ waveguide mode cannot be matched. This is overcome by tapering the waveguide along the propagation direction, which forces the optical mode of the waveguide to expand, thereby gradually transferring the mode from GaAs with high refractive index $\left(n_{\mathrm{GaAs}}=3.4\right)$ to air. The waveguide is tapered nonlinearly, as shown in Fig. 4(a), in order to achieve the required mode diameter at a working distance of a lensed fiber. This ensures that the fiber can be operated at a safe distance from the nanophotonic waveguide during experiments.

Figure 4(b) shows the effective index of the GaAs waveguide and the mode overlap as a function of waveguide width. The maximal mode overlap is achieved when the waveguide mode is almost entirely in air while still being guided, i.e., the effective index of the waveguide is close to 1 . For a waveguide width of $118 \mathrm{~nm}$, which can be fabricated, the mode overlap is maximized to $80 \%$. Efficient mode conversion in the present case from a 300 - to a 118 -nm waveguide requires that the adiabatic condition is fulfilled [49]:

$$
\frac{d w(z)}{d z} \ll n_{\text {eff }}(w)-n_{\text {clad }},
$$

where $n_{\text {eff }}\left(n_{\text {clad }}\right)$ is the effective index of the waveguide mode (the material surrounding the waveguide). That is, the width of the waveguide along the propagation direction $w(z)$ has to 
change slowly to prevent coupling of the fundamental mode to higher-order or counterpropagating modes. Because of the high $n_{\text {eff }}$ for GaAs waveguides, the effective index of the $300-\mathrm{nm}$ waveguide is relatively large compared to the refractive index of air, allowing us to change the width of the waveguide relatively fast initially. We define the adiabatic factor $\alpha$ from the relation

$$
\frac{d w(z)}{d z}=\alpha^{-1}\left[n_{\mathrm{eff}}(w)-1\right],
$$

where $\alpha \gg 1$ is the adiabatic condition. Setting $\alpha=1$ at first allows us to find the optimal taper shape, which can then be scaled afterwards to an arbitrary length that fulfills the adiabatic criterion. Equation (3) cannot be integrated directly because of the dependence of $n_{\mathrm{eff}}(w)$, so instead the change in position $\Delta z_{i}$ between two consecutive waveguide widths $w_{i}$ is obtained through $\Delta z_{i}=\Delta w /\left[n_{\mathrm{eff}}\left(w_{i}\right)-1\right]$. This equation defines the profile of the waveguide tapered from 300 to $118 \mathrm{~nm}$ as shown in Fig. 4(a). The length of the taper is finally $\alpha \sum \Delta z_{i}$, which is $1.8 \mu \mathrm{m}$ for $\alpha=1$. The taper fabricated on device $\mathrm{A}$ used in the quasiresonant HOM experiments in the paper was designed with $\alpha=10$. Below, we discuss the efficiency characterization results on another device (device B) with $\alpha=4$.

\section{B. Efficiency characterization: Device B}

The single-photon source efficiency has been thoroughly characterized on device B similar to device A in Fig. 1(a) but with no electrical contacts. It was fabricated on an intrinsic 160-nm-thick GaAs membrane and contains several waveguide sections with a photonic-crystal mirror termination on one side and waveguide taper on the other side, making the device unidirectional. The photonic-crystal waveguide has a $5-\mu \mathrm{m}-$ long slow-light section to enhance the light-matter coupling to reach a near unity $\beta$ factor [19]. The photonic-crystal waveguide is coupled to a 5- $\mu \mathrm{m}$-long straight nanobeam waveguide, eventually tapered to a width of $118 \mathrm{~nm}$ with an adiabatic parameter of $\alpha=4$ according to the design introduced above.

The overall photon-extraction efficiency of device $B$ is characterized by recording the total number of detected single photons on an avalanche photodiode (APD) from a QD in the photonic crystal section. A maximum count rate of $\sim 2 \mathrm{MHz}$ is obtained above saturation (see Fig. 5). The corresponding detected single-photon efficiency near the saturation power is $\sim 1 \mathrm{MHz}$ after correcting for the multiphoton probability reflected in the final value of $g^{2}(0)$ shown in Fig. 5. The multiphoton events stem from the emission of other QDs. This corresponds to an overall source efficiency of $\eta_{\mathrm{sp}}=10.3 \%$, where $\eta_{\mathrm{sp}}$ is the probability that upon excitation the QD emits a photon that is collected by the waveguide and subsequently successfully transferred into the optical fiber. The source efficiency is obtained by accounting for all propagation losses in the fiber and detection, including APD detection efficiency (26\%), spectral filtering efficiency $(30 \%)$, and the use of two mating sleeves in between fibers (79\% each). The source efficiency is limited by a finite preparation efficiency of the QD, propagation loss in the waveguide, and the coupling efficiency off chip. A thorough analysis of the source efficiency is presented in Ref. [50]. It may be further improved by implementing evanescent coupling from the waveguide to the fiber [50].
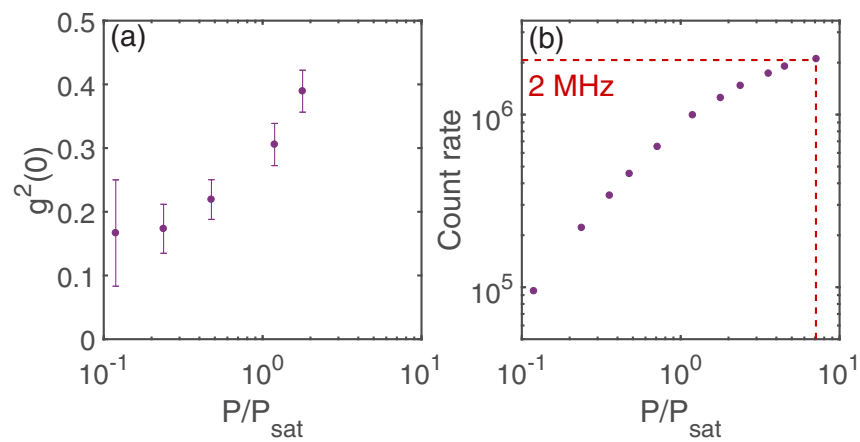

FIG. 5. Efficiency of a single-photon source with no electrical gates (device B). (a) Autocorrelation function at zero time delay $g^{2}(0)$ vs excitation power measured in units of the saturation power $P_{\text {sat }}$ (b) Raw count rate on an avalanche photodiode as a function of excitation power. A count rate of up to $2 \mathrm{MHz}$ is observed, where about half of it stems from the QD.

\section{TWO-PHOTON INTERFERENCE UNDER RESONANT EXCITATION}

\section{A. Structure design and experimental setup: Device C}

To experimentally test the influence of photon jitter, we have also carried out HOM measurements on QDs in a nanophotonic waveguide terminated with a grating outcoupler [device $C$; see Fig. 1(e)]. The wafer material and the fabrication procedure of the structure and the electrical contacts are the same as for device A [see Fig. 1(c) for the layer structure]. In the resonance fluorescence experiment, device $\mathrm{C}$ is mounted in a closedcycle cryostat and cooled to $1.58-1.7 \mathrm{~K}$, and the photons are collected from the top through an objective with $\mathrm{NA}=0.82$. The QD is excited by a tunable cw diode laser (Toptica CTL) polarized perpendicular to the waveguide that is tuned into resonance with the QD emission frequency. A forward bias of $0.1952 \mathrm{~V}$ is applied across the $p-i-n$ junction, resulting in an emission frequency of $v=325.457 \mathrm{THz}$. The residual laser is suppressed by the spatial separation between the QD and the collection spot alone as the polarization of the output grating is colinear with the laser polarization. A laser extinction relative to the resonant fluorescence intensity of more than 200 times is achieved under cw excitation. For the pulsed resonant HOM measurement, the QD is excited with 100-ps-long pulses created by electro-optical modulation of the diode laser at a rate of $72.6 \mathrm{MHz}$. Due to a finite modulation extinction, a constant cw laser contribution is present during the measurements. The $p$-shell excitation is achieved with a Ti:sapphire laser delivering picosecond-long pulses at a repetition rate of $76 \mathrm{MHz}$. The HOM interferometer used for the measurements with both excitation schemes is similar to the one sketched in Fig. 1(f), now with a time delay of $\Delta t=13.8 \mathrm{~ns}$ and $R=0.5$ and $T=0.5$. In addition to the $70-\mathrm{pm} / 25-\mathrm{GHz}$ grating filter ( $72 \%$ transmission) the photons are tightly filtered by an etalon with a bandwidth of $3 \mathrm{GHz}$ ( $90 \%$ transmission).

\section{B. Quasiresonant excitation and spectral filtering}

The implementation of spectral filtering offers one approach to improve indistinguishability. Figure 6 shows a pulsed HOM interference measurement for the $\mathrm{QD}$ in device $\mathrm{C}$ 


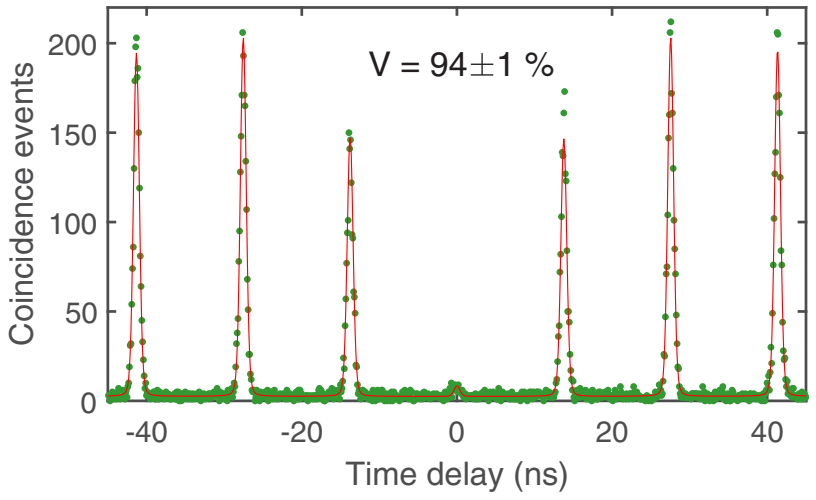

FIG. 6. Coincidences histogram of the HOM interference data recorded on device $\mathrm{C}$ under quasiresonant excitation for a successive time separation of emitted photons of $\sim 13 \mathrm{~ns}$, measured at $1.7 \mathrm{~K}$ and at $P_{\text {sat }}$. The data points present the raw data without any background correction. The fit of the experimental data to theory is shown by the red line.

recorded under $p$-shell excitation and by implementing a 3-GHz bandpass etalon filter. We observe a degree of indistinguishability as high as $94 \pm 1 \%$, which approaches the fundamental limit set by the phonon broadening in nanobeam waveguides [32]. This value could potentially be improved even further by implementing rate enhancement, either through the Purcell effect or through a large oscillator strength. For comparison, we observe an indistinguishability of $77 \%$ for this QD in the absence of spectral filtering, where the observed reduction could be partly due to the minor neighboring peak observed in Fig. 8.

\section{Resonant excitation}

Strict resonant excitation may be implemented in order to achieve high indistinguishability without spectral filtering. Figures 7(a) and 7(b) show the results of HOM measurements using 100-ps pulsed excitation and cw excitation, respectively. The high degree of indistinguishability is immediately visible in the pulsed data by the complete absence of a peak around

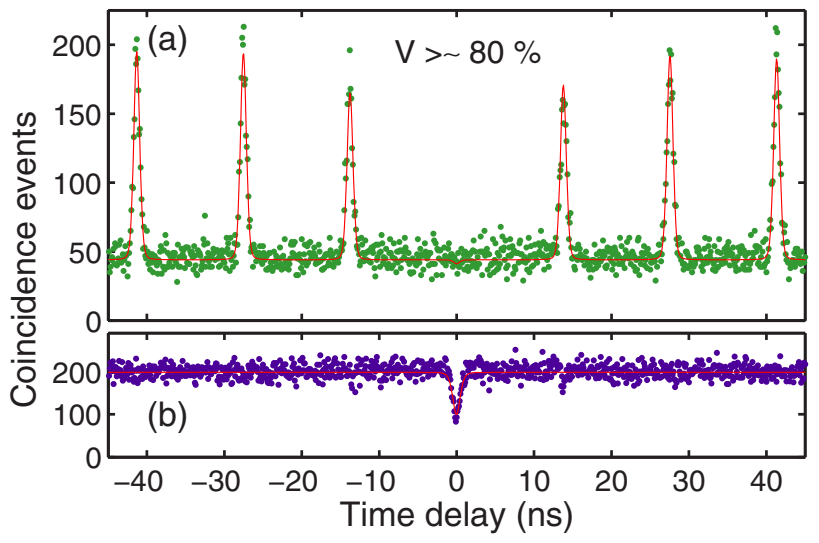

FIG. 7. HOM correlation histograms of device $\mathrm{C}$ in the case of strict resonant excitation with 100 -ps-long pulses at $0.8 P_{\text {sat }}$ (a) and with continuous-wave excitation at $0.5 P_{\text {sat }}$ (b). The operation temperature of the experiment is $1.58 \mathrm{~K}$. The fit of the experimental data to theory is shown by the red line.

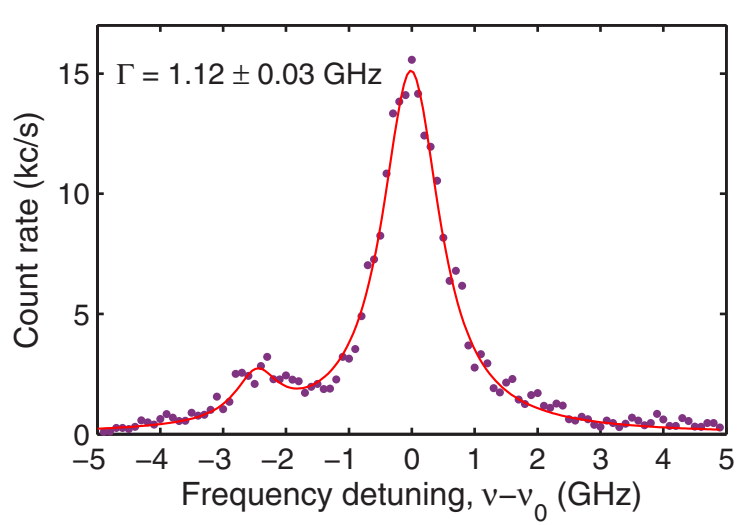

FIG. 8. Resonance fluorescence of a quantum dot vs laser frequency detuning. The data are recorded at a power of $0.2 P_{\text {sat }}$ at $1.58 \mathrm{~K}$. The resonance frequency $v=325.457 \mathrm{THz}$ corresponds to a wavelength of $921.14 \mathrm{~nm}$. The solid line is a Lorentzian fit to the data with linewidth $\Gamma=1.12 \pm 0.03 \mathrm{GHz}$. The weak peak on the lower-energy side stems from another QD dipole weakly coupled to the waveguide.

zero time delay. However, in this case a quantitative extraction of a degree of indistinguishability from the pulsed data is complicated by two effects: the applied pulsed laser source had a cw background (at the level of 23\%) and the relatively long excitation pulses imply that a double excitation of the QD occurs with a non-negligible probability [leading to a contribution $g^{2}(0) \sim 5.5 \%$ ]. The former effect leads to a finite background level in the data of Fig. 7(a), which has the temporal dependence of the data in Fig. 7(b). In the present paper we attempt to include these background effects (see Appendix B for the details of the analysis), and we conclude that the degree of indistinguishability is $\gtrsim 80 \%$. We note that this is a conservative estimate since in the analysis it is assumed that the background originates solely from the $\mathrm{cw}$ fluorescence from the QD, while the finite extinction of the pump laser would provide a flat background and therefore lead to a higher extracted indistinguishability. From the resonant-excitation experiment, by scanning the laser frequency across the QD resonance and recording the integrated intensity on a $\mathrm{CCD}$ camera, we also extract a QD linewidth of $1.12 \mathrm{GHz}$ (see Fig. 8), which is only 1.3 times wider than the natural linewidth. We emphasize that a transform-limited linewidth is a much stricter requirement than the demonstration of subsequently emitted indistinguishable photons, since the former is sensitive to slow charge noise [20]. This confirms the expectation that the extracted degree of indistinguishability of $\gtrsim 80 \%$ is a conservative lower bound. Achieving a narrow linewidth is essential for fully exploiting the high cooperativity of the photon-QD interface [12].

\section{SUMMARY}

In conclusion, we have experimentally demonstrated highpurity and highly indistinguishable single-photon sources based on QDs embedded in planar nanoscale waveguides with integrated electrical contacts. The role of photon jitter in quasiresonant excitation was identified, and a significant improvement in indistinguishability was observed when implementing spectral filtering or strict resonant excitation. A high indistinguishability of $\sim 94 \%$ was found, which illustrated 
the exciting potential of the planar waveguide platform. The indistinguishability can readily be further improved by enhancing the decay rate of the QD, which can be done by increasing the oscillator strength of the QD [51] or by implementing even modest Purcell enhancement [42]. Alternatively the temperature could be reduced further or the relevant decoherence phonon modes suppressed [32]. A major issue for resonant excitation schemes is to efficiently extinguish the excitation laser from the signal, and here the planar platform may be advantageous since the pump beam could be guided laterally through the structure [52] or a vertical pump beam may be applied at one spot while collection is from another. This may overcome the intrinsic source efficiency limitations of $25 \%$ found in vertical devices when implementing cross-polarization extinction in excitation [22-25]. Combining these functionalities into a single device would eventually enable a fully deterministic and coherent single-photon source, which could subsequently be spatially demultiplexed by implementing fast switches to generate a scalable resource of single photons. The limit to the number of achievable simultaneous photons on demand with such an approach is ultimately determined by any loss processes that lead to an exponential reduction of the rate of photon generation. This constitutes an important future engineering challenge. Another important application area is for the development of deterministic spin-photon interfaces, where coherent light-matter interaction is a prerequisite for advanced quantum-network architectures [53].

\section{ACKNOWLEDGMENTS}

We acknowledge K. Prindal-Nielsen for the $\beta$ factor calculations, C. Papon for the help with the characterization of the single-photon source efficiency, L. Zhai for the $g^{2}(0)$ calculations, and T. Schröder for valuable input on the experimental setup. We gratefully acknowledge financial support from the European Research Council (Advanced Grant "SCALE"), Innovation Fund Denmark (Quantum Innovation Center "Qubiz"), and the Danish Council for Independent Research. S.I.P. and J.D.S. acknowledge support from the KIST flagship institutional program. A.V.K., I.S., M.L., and R.J.W. acknowledge support from Swiss National Science Foundation (SNF) (Project No. 200020_156637) and National Centres of Competence in Research (NCCR) QSIT. A.L. and A.D.W. gratefully acknowledge the support of The Federal Ministry of Education and Research (BMBF) Q.com-H Grant No. 16KIS0109 and Deutsche Forschungsgemeinschaft Grant No. TRR 160.

\section{APPENDIX A: RELIABLE EXTRACTION OF PHOTON INDISTINGUISHABILITY FROM A PULSED HONG-OU-MANDEL EXPERIMENT}

In this section, we present the fitting routine used to model the Hong-Ou-Mandel data and demonstrate the key importance of applying appropriate noise statistics for the coincidence counts and the correct line shape for the peaks in order to reliably extract the photon indistinguishability.

The pulsed two-photon correlation histograms in Figs. 3(a) and 3(b) consist of a series of peaks. The peak amplitudes are determined by the relative probabilities for two photons to propagate along the different paths of a Mach-Zehnder interferometer, with either 2.7- or 13-ns delay lengths. The central peak near zero time delay $\Delta t=0$ is the coincidence counts for two photons arriving on the two APDs after interfering on the last beam splitter. For completely indistinguishable photons the central amplitude is zero and the degree of indistinguishability can be quantified as the amplitude relative to the expected amplitude obtained for distinguishable photons.

As seen in the data in Fig. 3(a) for a path difference of $2.7 \mathrm{~ns}$ the correlation histograms consist of a five-peak cluster repeated with a 13-ns period. The visibility in this case is given by [22]

$$
V_{2.7 \mathrm{~ns}}=\frac{R^{2}+T^{2}}{2 R T}-\frac{2 A_{0}}{A_{+2.7 \mathrm{~ns}}+A_{-2.7 \mathrm{~ns}}},
$$

where $A_{0}$ is the area of the central peak and $A_{ \pm 2.7 \mathrm{~ns}}$ is the area of the two neighboring peaks with $R=0.46$ and $T=$ 0.54 being the reflectivity and transmission of the last beam splitter. We note that no correction for the small $g^{2}(0)<0.006$ is implemented in the analysis since it is an intrinsic property of the photon source and not the measurement apparatus. For the path difference of $13 \mathrm{~ns}$ the correlation histograms result in a set of peaks each separated by the inverse of the laser repetition rate of $13 \mathrm{~ns}$. The visibility is here given as [23]

$$
V_{13 \mathrm{~ns}}=\frac{R^{2}+T^{2}-A_{0} / A}{2 R T},
$$

where $A$ is the average amplitude of peaks $25 \mathrm{~ns}$ or further away.

The five-peak cluster for 2.7-ns path difference consists of overlapping peaks resulting from the exponential QD decay with a finite rate that is on the order of the time separation between the excitation pulses. The coincidence counts therefore never reach zero, even for perfect indistinguishability, and the tail of the neighboring peaks strongly influences the fitted amplitudes. Such data must therefore be modeled carefully in order to extract reliable values of the photon indistinguishability.

We implement a rigorous fitting routine, which takes into account the exponential decay of the emitter, the measured instrument response function (IRF), and Poissonian counting statistics. Each peak is modeled as a double-sided single exponential decay convoluted with the measured IRF. The repetition rate of the laser and the decay rate of the QD is obtained independently from the time-resolved measurements. Likewise, the ratio between pairs of neighboring peaks is fixed by the measured beam splitter transmission and reflection. The remaining free parameters are the individual peak amplitudes, the Mach-Zehnder time delay, an overall time shift, and a background. The IRF is measured by sending a laser pulse through the detection setup and the resulting peaks are fitted with a Voigt function and a background to obtain a backgroundfree IRF used in the convolution. The Voigt function accounts for a longer tail present in the measured IRF that is not captured by a Gaussian fit (see Fig. 9).

We note that the photon indistinguishability data (see Fig. 3) only contain few coincidence counts in time bins in between the peaks and, more importantly, in the central peak, which contains the information about the indistinguishability. These 


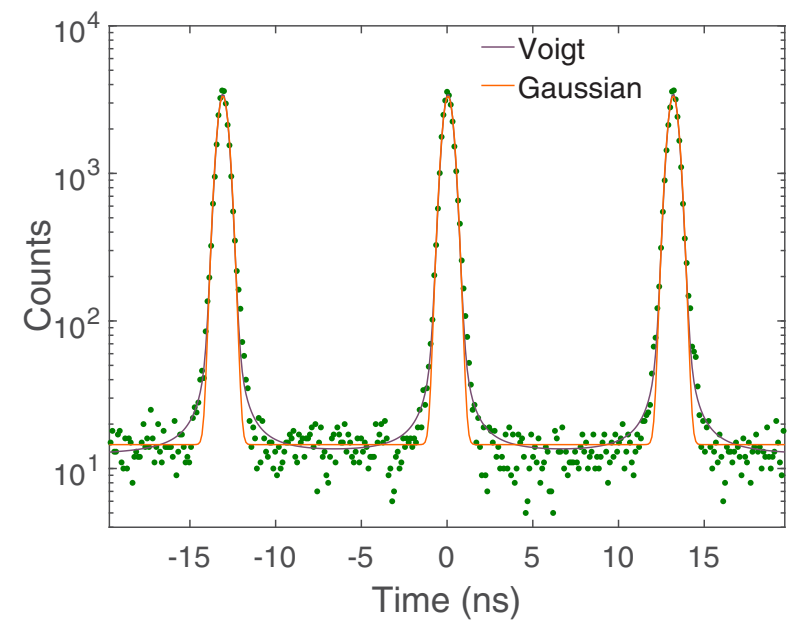

FIG. 9. The measured instrument response function (IRF) of the Hanbury Brown and Twiss setup fitted with both a Voigt (purple) and a Gaussian (orange) function using the Poisson MLE described in the text. The Voigt function with its broader tail provides a superior fit to the line shape of the IRF evidenced by the smaller $\chi_{\text {MLE }}^{2}=2.3$ vs 5.7 for the Gaussian fit.

low counts pose a challenge for traditional least-square-fitting routines that minimizes the reduced $\chi_{\text {red }}^{2}$ :

$$
\chi_{\mathrm{red}}^{2}=\frac{1}{n-v} \sum_{i=0}^{n} \frac{\left[y_{i}-f\left(\Delta t_{i}\right)\right]^{2}}{y_{i}},
$$

where $n$ is the number of data points and $v$ is the number of free parameters. Here $y_{i}$ is the measured data point and $f_{i} \equiv f\left(\Delta t_{i}\right)$ is the fitting function associated with the data bin $\Delta t_{i}$. Minimizing Eq. (A3) assumes a Gaussian noise distribution whereas the coincidence counts in reality follow a Poisson distribution [54]. Using traditional least-square-fitting procedures on such data is therefore known to lead to biases [55] where especially bins with $y_{i}=0$ cannot be properly handled as they cause $\chi^{2}$ to diverge.

To overcome the problem of low counts we instead use the maximum likelihood estimator (MLE) for the Poisson distribution and optimize that over the model parameters. The probability of measuring $y_{i}$ coincidence events in the time bin $\Delta t_{i}$ when expecting $f_{i}$ on average is given by the Poisson distribution

$$
P\left(y_{i} \mid f_{i}\right)=\frac{f_{i}^{y_{i}}}{y_{i} !} \exp -f_{i} .
$$

Assuming independent data points the global likelihood is

$$
\mathcal{L}(\mathbf{y} \mid \mathbf{f})=\prod_{i=1}^{n} P\left(y_{i} \mid f_{i}\right) .
$$

To overcome underflow errors it is customary to minimize twice the negative logarithm of the normalized likelihood, i.e.,

$$
\begin{aligned}
\chi_{\text {mle }}^{2} & =-2 \ln \left(\frac{\mathcal{L}(\mathbf{y} \mid \mathbf{f})}{\mathcal{L}(\mathbf{y} \mid \mathbf{y})}\right) \\
& =2 \sum_{i=1}^{n}\left(f_{i}-y_{i}\right)-2 \sum_{\substack{i=1 \\
y_{i} \neq 0}}^{n} x_{i} \ln \left(f_{i} / y_{i}\right) .
\end{aligned}
$$
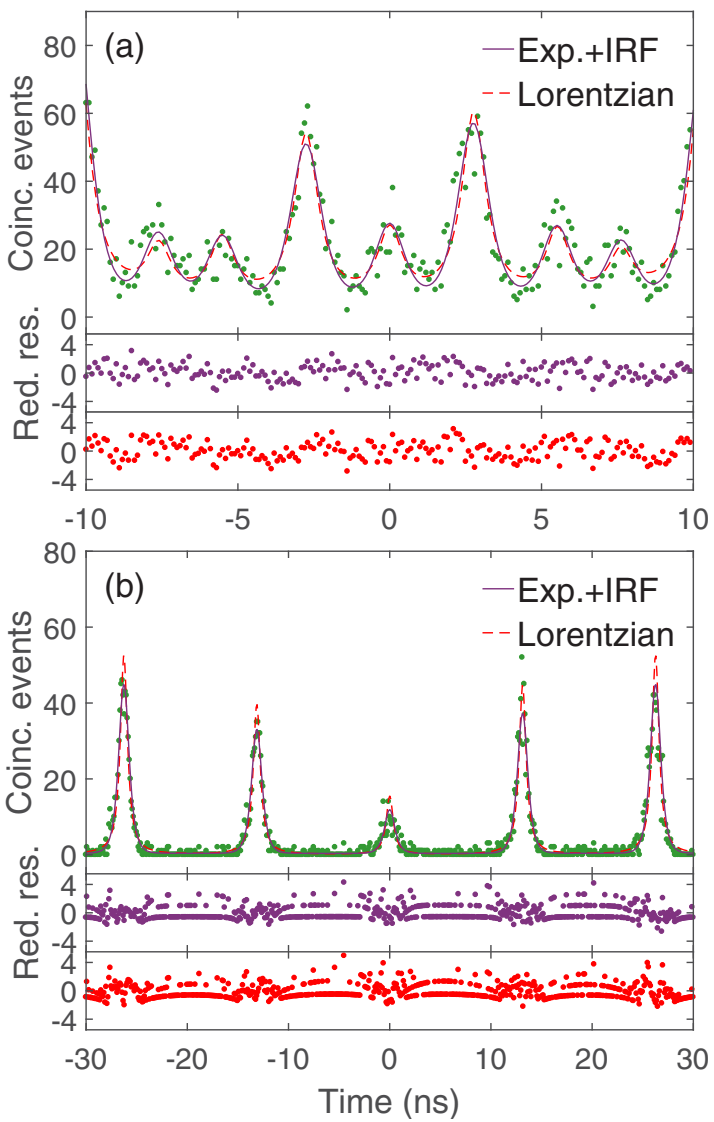

FIG. 10. Two-photon coincidence data at $4.2 \mathrm{~K}$ for $2.7-n s$ (a) and 13-ns (b) path delay. The peaks are fitted with either double-sided exponentials convoluted with the measured instruments response (solid purple) or a Lorentzian (dashed red) by optimizing the Poisson MLE. The corresponding reduced residues for each fit are shown below the figures.

Similarly to the reduced $\chi_{\text {red }}^{2}$ for least-square fitting, the figure of merit $\chi_{\text {mle }}^{2}$ approaches one after normalizing with the number of degrees of freedom $n-v$ when the model accurately describes the data. In the data analysis the global minimum optimization is run 50 times with a random set of initial parameters to ensure proper convergence since the algorithm is less robust than the Levenberg-Marquardt routine used for least-square fitting.

Another important point is to choose the correct line shape of the correlation function to model the data. We compare in Table I the extracted values for the two-photon-interference visibility $V$ and the reduced $\chi_{\text {mle }}^{2}$ when applying the correct exponential line shape and measurement IRF compared to the case of a heuristic Lorentzian function. The "long tail" of the Lorentzian function essentially implies that the extracted visibility $V$ is systematically and significantly overestimated in this case. The corresponding fits to the experimental data are reproduced in Fig. 10(a). Table I summarizes the outcome of the detailed analysis of our indistinguishability data with the different approaches applied in the literature.

For the 13-ns time delay we again compare our data with Lorentzian peaks as shown in Fig. 10(b). For all the datasets the Lorentzian functions overestimate the peak heights and 
TABLE I. Comparison of the fitting procedure used to analyze the data of the Hong-Ou-Mandel experiment when the peaks are fitted by either double-sided exponentials convoluted with the instrument response (Exp \& IRF/MLE) or Lorentzians (Lorz). The quoted error bars are $95 \%$ confidence intervals for the parameters extracted from the fits. The reported $\chi_{\text {mle }}^{2}$ values are normalized by $1 /(n-v)$. Also shown are literature references using the presented fitting routines to extract the HOM visibility in QDs. Lorz, Lorentzian functions; LS, least square; MLE, maximum likelihood estimator.

\begin{tabular}{|c|c|c|c|c|c|c|}
\hline \multicolumn{7}{|c|}{$\Delta t=2.7 \mathrm{~ns}$} \\
\hline \multirow[b]{2}{*}{$T(\mathrm{~K})$} & \multicolumn{2}{|c|}{ Exp \& IRF/MLE } & \multicolumn{2}{|c|}{ Lorz/LS } & \multicolumn{2}{|c|}{ Exp \& IRF/LS } \\
\hline & Visibility $V$ & $\overline{\chi_{\mathrm{mle}}^{2}}$ & Visibility $V$ & $\chi_{\mathrm{red}}^{2}$ & Visibility $V$ & $\chi_{\mathrm{red}}^{2}$ \\
\hline 4.2 & $0.62 \pm 0.02$ & 1.29 & $0.75 \pm 0.04$ & 1.82 & $0.65 \pm 0.03$ & 1.42 \\
\hline 10 & $0.54 \pm 0.02$ & 1.29 & $0.64 \pm 0.05$ & 1.82 & $0.56 \pm 0.04$ & 1.46 \\
\hline 15 & $0.40 \pm 0.02$ & 1.47 & $0.50 \pm 0.05$ & 1.83 & $0.43 \pm 0.04$ & 1.62 \\
\hline 20 & $0.20 \pm 0.02$ & 2.04 & $0.27 \pm 0.05$ & 2.00 & $0.23 \pm 0.04$ & 2.28 \\
\hline 25 & $0.21 \pm 0.04$ & 1.31 & $0.24 \pm 0.07$ & 1.67 & $0.22 \pm 0.06$ & 1.53 \\
\hline Methods in literature & [43] & & {$[21,26,40]$} & & {$[22,25,39,41,42]$} & \\
\hline
\end{tabular}

at the same time predict a too broad tail. As evident from the small reduced residues the data points above the fitted peaks for exponential fit are within the expected error bars for Poissonian noise. The overestimated central peak leads to overall lower visibilities, despite the overestimated side peaks, and the broad tail causes the background to be pushed below zero. The latter is not physical since only positive values are allowed in the Poisson distribution.

\section{APPENDIX B: ANALYSIS OF THE TWO-PHOTON CORRELATION HISTOGRAM UNDER RESONANT EXCITATION WITH CONTINUOUS-WAVE BACKGROUND}

The measured two-photon correlation histogram under strict resonant excitation is shown in Fig. 7, and it is observed that the peak near zero time delay is virtually absent. However, an overall background arises from coincidence counts from photons emitted when the QD is excited by the residual $\mathrm{cw}$ laser. The background complicates the analysis of the indistinguishability measurements. Most importantly the cw HOM coincidence histogram exhibits a trough at zero time delay that masks the true peak height in the pulsed HOM histogram, which needs to be accounted for. This trough in the data cancels the residual peak in the pulsed indistinguishability measurement. For an infinitely fast detector the trough would go to zero for a single QD, i.e., the depth and the width of the measured trough [Fig. 7(b)] depend on the time response of the system, the lifetime, the coherence time, and $g^{2}(0)$ [56].

To estimate the peak amplitude at zero time delay $\tilde{A}_{0}$, we may subtract the trough depth, obtained from the cw HOM measurements. An estimate of the indistinguishability $\tilde{V}$ can then be obtained from Eq. (A2) by using the average amplitude of peaks at longer delay in combination with $\tilde{A}_{0}$. Hence, using the peak/trough area as done in the previous section would significantly underestimate the indistinguishability, since the $\mathrm{cw}$ trough is wider than the peaks in the pulsed measurement. Instead we use the amplitudes of the peaks to extract $\tilde{V}$.

To estimate the trough depth $A_{\mathrm{cw}}$ the cw HOM data are modeled with a double-sided exponential trough convoluted with the measured instrument response function of the full system, $f_{\mathrm{cw}}(t)=A_{\mathrm{cw}}\left[1-\exp \left(-\gamma_{\mathrm{cw}}|\Delta t|\right)\right] \otimes \operatorname{IRF}(\Delta \mathrm{t})$, where the fitted rate is $\gamma_{\mathrm{cw}}=3.1 \pm 0.2 \mathrm{~ns}^{-1}$. This functional form is an approximation to the real shape but is sufficient to extract the trough depth and it limits the number of free parameters.

The pulsed HOM data are modeled with a series of doublesided exponents convoluted with the instrument response, $f_{\mathrm{P}}(t)$, as described in Appendix $\mathrm{A}$, in addition to a background $A_{\text {bg }}=44.0$. Since the data may have a trough from the cw contribution we allow for the central peak of the fit to have a negative amplitude. The decay rate is fitted to be $\gamma_{\mathrm{P}}=6.2 \pm 0.2 \mathrm{~ns}^{-1}$, which is a free parameter since the lifetime of the QD is IRF limited. From these data we can use the peak amplitudes and backgrounds to extract the central peak and side peak amplitudes

$$
\begin{aligned}
& \tilde{A}_{0}=f_{\mathrm{P}}(0)-f_{\mathrm{cw}}(0) \frac{A_{\mathrm{bg}}}{A_{\mathrm{cw}}}=18.9, \\
& \tilde{A}=f_{\mathrm{P}}(2 \Delta t)=148.9,
\end{aligned}
$$

and from Eq. (A2) we get an estimate of the indistinguishability of $\tilde{V} \sim 75 \%$.

We emphasize that the above estimate is a lower boundary of the actual indistinguishability. Hence, the 100-ps-long excitation pulses imply that there is a finite probability to re-excite the QD since it may have emitted a photon before the excitation pulse is over. This effect leads to a nonzero $g^{2}(0)$ and increases the central peak height in the pulsed HOM measurement. This additional contribution to $g^{2}(0)$ is a consequence of the excitation scheme and not intrinsic to the source. We account for it by including a correction for a finite $g^{2}(0)$ in the expression for the two-photon interference visibility in Eq. (A2). Following the procedure in Ref. [23] for expressing the expected peaks area and adding a correction factor $1+g^{2}(0)$ for the central peak area similarly to Ref. [13], we have

$$
\tilde{A_{0}} / \tilde{A}=\left(R^{2}+T^{2}\right)\left[1+g^{2}(0)\right]+2 R T V,
$$

and we get a corrected expression for the two-photon interference visibility:

$$
\tilde{V}_{13 \mathrm{~ns}}=\frac{\left(R^{2}+T^{2}\right)\left[1+g^{2}(0)\right]-\tilde{A}_{0} / \tilde{A}}{2 R T} .
$$


The residual $g^{2}(0)$ due to re-excitation can be calculated numerically by including also the response of the applied etalon to spectrally filter the emitted photons. We find $g^{2}(0)$ $\sim 0.055$. Using this value in Eq. (B3), we estimate that the indistinguishability of the photons obtained by resonant pulsed excitation is $\tilde{V}_{13 \mathrm{~ns}} \gtrsim 80 \%$.
[1] E. Diamanti, H.-K. Lo, B. Qi, and Z. Yuan, NPJ Quant. Info. 2, 16025 (2016).

[2] N. Sangouard and H. Zbinden, J. Mod. Opt. 59, 1458 (2012).

[3] A. Aspuru-Guzik and P. Walther, Nat. Phys. 8, 285 (2012).

[4] P. Kok, W. J. Munro, K. Nemoto, T. C. Ralph, J. P. Dowling, and G. J. Milburn, Rev. Mod. Phys. 79, 135 (2007).

[5] T. Rudolph, APL Photon. 2, 030901 (2017).

[6] H. J. Kimble, Nature (London) 453, 1023 (2008).

[7] P. Lodahl, Quantum Sci. Technol. (2017), doi:10.1088/20589565/aa91bb.

[8] X.-L. Wang, L.-K. Chen, W. Li, H.-L. Huang, C. Liu, C. Chen, Y.-H. Luo, Z.-E. Su, D. Wu, Z.-D. Li, H. Lu, Y. Hu, X. Jiang, C.-Z. Peng, L. Li, N.-L. Liu, Y.-A. Chen, C.-Y. Lu, and J.-W. Pan, Phys. Rev. Lett. 117, 210502 (2016).

[9] J. B. Spring, B. J. Metcalf, P. C. Humphreys, W. S. Kolthammer, X.-M. Jin, M. Barbieri, A. Datta, N. Thomas-Peter, N. K. Langford, D. Kundys, J. C. Gates, B. J. Smith, P. G. R. Smith, and I. A. Walmsley, Science 339, 798 (2013).

[10] M. A. Broome, A. Fedrizzi, S. Rahimi-Keshari, J. Dove, S. Aaronson, T. C. Ralph, and A. G. White, Science 339, 794 (2013).

[11] P. Michler, Science 290, 2282 (2000).

[12] P. Lodahl, S. Mahmoodian, and S. Stobbe, Rev. Mod. Phys. 87, 347 (2015)

[13] C. Santori, D. Fattal, J. Vučković, G. S. Solomon, and Y. Yamamoto, Nature (London) 419, 594 (2002).

[14] S. Laurent, S. Varoutsis, L. Le Gratiet, A. Lemaitre, I. Sagnes, F. Raineri, A. Levenson, I. Robert-Philip, and I. Abram, Appl. Phys. Lett. 87, 163107 (2005).

[15] T. Lund-Hansen, S. Stobbe, B. Julsgaard, H. Thyrrestrup, T. Sünner, M. Kamp, A. Forchel, and P. Lodahl, Phys. Rev. Lett. 101, 113903 (2008).

[16] J. Claudon, J. Bleuse, N. S. Malik, M. Bazin, P. Jaffrennou, N. Gregersen, C. Sauvan, P. Lalanne, and J.-M. Gérard, Nat. Photon. 4, 174 (2010).

[17] J. H. Prechtel, A. V. Kuhlmann, J. Houel, L. Greuter, A. Ludwig, D. Reuter, A. D. Wieck, and R. J. Warburton, Phys. Rev. X 3, 041006 (2013).

[18] A. V. Kuhlmann, J. Houel, A. Ludwig, L. Greuter, D. Reuter, A. D. Wieck, M. Poggio, and R. J. Warburton, Nat. Phys. 9, 570 (2013).

[19] M. Arcari, I. Söllner, A. Javadi, S. L. Hansen, S. Mahmoodian, J. Liu, H. Thyrrestrup, E. H. Lee, J. D. Song, S. Stobbe, and P. Lodahl, Phys. Rev. Lett. 113, 093603 (2014).

[20] A. V. Kuhlmann, J. H. Prechtel, J. Houel, A. Ludwig, D. Reuter, A. D. Wieck, and R. J. Warburton, Nat. Commun. 6, 8204 (2015).

[21] M. Gschrey, A. Thoma, P. Schnauber, M. Seifried, R. Schmidt, B. Wohlfeil, L. Krüger, J.-H. Schulze, T. Heindel, S. Burger, F. Schmidt, A. Strittmatter, S. Rodt, and S. Reitzenstein, Nat. Commun. 6, 7662 (2015).

[22] N. Somaschi, V. Giesz, L. de Santis, J. C. Loredo, M. P. Almeida, G. Hornecker, S. L. Portalupi, T. Grange, C. Antón, J. Demory, C. Gómez, I. Sagnes, N. D. Lanzillotti-Kimura, A. Lemaítre, A.
Auffeves, A. G. White, L. Lanco, and P. Senellart, Nat. Photon. 10, 340 (2016).

[23] J. C. Loredo, N. A. Zakaria, N. Somaschi, C. Anton, L. de Santis, V. Giesz, T. Grange, M. A. Broome, O. Gazzano, G. Coppola, I. Sagnes, A. Lemaitre, A. Auffeves, P. Senellart, M. P. Almeida, and A. G. White, Optica 3, 433 (2016).

[24] H. Wang, Z.-C. Duan, Y.-H. Li, S. Chen, J.-P. Li, Y.-M. He, M.-C. Chen, Y. He, X. Ding, C.-Z. Peng, C. Schneider, M. Kamp, S. Höfling, C.-Y. Lu, and J.-W. Pan, Phys. Rev. Lett. 116, 213601 (2016).

[25] X. Ding, Y. He, Z.-C. Duan, N. Gregersen, M.-C. Chen, S. Unsleber, S. Maier, C. Schneider, M. Kamp, S. Höfling, C.-Y. Lu, and J.-W. Pan, Phys. Rev. Lett. 116, 020401 (2016).

[26] A. Thoma, P. Schnauber, M. Gschrey, M. Seifried, J. Wolters, J.-H. Schulze, A. Strittmatter, S. Rodt, A. Carmele, A. Knorr, T. Heindel, and S. Reitzenstein, Phys. Rev. Lett. 116, 033601 (2016).

[27] J. Houel, A. V. Kuhlmann, L. Greuter, F. Xue, M. Poggio, B. D. Gerardot, P. A. Dalgarno, A. Badolato, P. M. Petroff, A. Ludwig, D. Reuter, A. D. Wieck, and R. J. Warburton, Phys. Rev. Lett. 108, 107401 (2012).

[28] S. Kalliakos, Y. Brody, A. Schwagmann, A. J. Bennett, M. B. Ward, D. J. P. Ellis, J. Skiba-Szymanska, I. Farrer, J. P. Griffiths, G. A. C. Jones, D. A. Ritchie, and A. Shields, Appl. Phys. Lett. 104, 221109 (2014).

[29] S. Kalliakos, Y. Brody, A. J. Bennett, D. J. P. Ellis, J. SkibaSzymanska, I. Farrer, J. P. Griffiths, D. A. Ritchie, and A. J. Shields, Appl. Phys. Lett. 109, 151112 (2016).

[30] L. Midolo, T. Pregnolato, G. Kiršanskè, and S. Stobbe, Nanotechnology 26, 484002 (2015).

[31] C. K. Hong, Z. Y. Ou, and L. Mandel, Phys. Rev. Lett. 59, 2044 (1987).

[32] P. Tighineanu, C. L. Dreeßen, C. Flindt, P. Lodahl, and A. S Sørensen, arXiv:1702.04812.

[33] P. W. Fry, I. E. Itskevich, D. J. Mowbray, M. S. Skolnick, J. J. Finley, J. A. Barker, E. P. O’Reilly, L. R. Wilson, I. A. Larkin, P. A. Maksym, M. Hopkinson, M. Al-Khafaji, J. P. R. David, A. G. Cullis, G. Hill, and J. C. Clark, Phys. Rev. Lett. 84, 733 (2000).

[34] D. M. Bruls, P. M. Koenraad, H. W. M. Salemink, J. H. Wolter, M. Hopkinson, and M. S. Skolnick, Appl. Phys. Lett. 82, 3758 (2003).

[35] S. Raymond, S. Studenikin, A. Sachrajda, Z. Wasilewski, S. J. Cheng, W. Sheng, P. Hawrylak, A. Babinski, M. Potemski, G. Ortner, and M. Bayer, Phys. Rev. Lett. 92, 187402 (2004).

[36] J. H. Blokland, F. J. P. Wijnen, P. C. M. Christianen, U. Zeitler, J. C. Maan, P. Kailuweit, D. Reuter, and A. D. Wieck, Phys. Rev. B 75, 233305 (2007).

[37] C. Santori, D. Fattal, J. Vučković, G. S. Solomon, E. Waks, and Y. Yamamoto, Phys. Rev. B 69, 205324 (2004).

[38] M. Davanço, C. S. Hellberg, S. Ates, A. Badolato, and K. Srinivasan, Phys. Rev. B 89, 161303 (2014). 
[39] Y.-M. He, Y. He, Y.-J. Wei, D. Wu, M. Atatüre, C. Schneider, S. Höfling, M. Kamp, C.-Y. Lu, and J.-W. Pan, Nat. Nanotechnol. 8, 213 (2013).

[40] O. Gazzano, S. M. de Vasconcellos, C. Arnold, A. Nowak, E. Galopin, I. Sagnes, L. Lanco, A. Lemaître, and P. Senellart, Nat. Commun. 4, 1425 (2013).

[41] K. H. Madsen, S. Ates, J. Liu, A. Javadi, S. M. Albrecht, I. Yeo, S. Stobbe, and P. Lodahl, Phys. Rev. B 90, 155303 (2014).

[42] T. Grange, N. Somaschi, C. Antón, L. de Santis, G. Coppola, V. Giesz, A. Lemaître, I. Sagnes, A. Auffèves, and P. Senellart, Phys. Rev. Lett. 118, 253602 (2017).

[43] B. Kambs, J. Kettler, M. Bock, J. N. Becker, C. Arend, A. Lenhard, S. L. Portalupi, M. Jetter, P. Michler, and C. Becher, Opt. Express 24, 22250 (2016).

[44] A. Kiraz, M. Atatüre, and A. Imamoglu, Phys. Rev. A 69, 032305 (2004).

[45] E. A. Muljarov and R. Zimmermann, Phys. Rev. Lett. 93, 237401 (2004).

[46] G. Reithmaier, F. Flassig, P. Hasch, S. Lichtmannecker, K. Müller, J. Vučković, R. Gross, M. Kaniber, and J. J. Finley, Appl. Phys. Lett. 105, 081107 (2014).

[47] M. Kroner, K. M. Weiss, S. Seidl, R. J. Warburton, A. Badolato, P. M. Petroff, and K. Karrai, Phys. Status Solidi 246, 795 (2009).
[48] J. D. Cohen, S. M. Meenehan, and O. Painter, Opt. Express 21, 11227 (2013).

[49] A. W. Snyder and J. D. Love, Optical Waveguide Theory, 1st ed. (Springer, Boston, 1984), p. 738.

[50] R. S. Daveau, K. C. Balram, T. Pregnolato, J. Liu, E. H. Lee, J. D. Song, V. Verma, R. Mirin, S. W. Nam, L. Midolo, S. Stobbe, K. Srinivasan, and P. Lodahl, Optica 4, 178 (2017).

[51] P. Tighineanu, R. S. Daveau, T. B. Lehmann, H. E. Beere, D. A. Ritchie, P. Lodahl, and S. Stobbe, Phys. Rev. Lett. 116, 163604 (2016).

[52] A. Muller, E. B. Flagg, P. Bianucci, X. Y. Wang, D. G. Deppe, W. Ma, J. Zhang, G. J. Salamo, M. Xiao, and C. K. Shih, Phys. Rev. Lett. 99, 187402 (2007).

[53] W. B. Gao, A. Imamoglu, H. Bernien, and R. Hanson, Nat. Photon. 9, 363 (2015).

[54] P. Gregory, Bayesian Logical Data Analysis for the Physical Sciences: A Comparative Approach with Mathematica Support (Cambridge University Press, Cambridge, England, 2005).

[55] T. A. Laurence and B. A. Chromy, Nat. Methods 7, 338 (2010).

[56] R. Proux, M. Maragkou, E. Baudin, C. Voisin, P. Roussignol, and C. Diederichs, Phys. Rev. Lett. 114, 067401 (2015). 